\title{
Lidars and Other Devices for Remote Analysis
}

DOI: $10.1134 / \mathrm{S} 106193480906001 \mathrm{X}$

Needs in remote chemical analysis are growing, which is due to the increasing number of hazardous production facilities, the necessity of controlling the air above the plants or in city streets, the development of space research, and so on. The problem of remote analysis is often solved using physical, particularly spectroscopic, methods, starting from the detection of helium in the Sun in the end of the 19th century. An important place among these methods should be occupied by lidars, laser spectroscopic instruments also intended for analysis at more or less significant distances. In any case, these instruments seem convenient for controlling air along traffic arteries or emissions from plant chimneys.

Lidars (LIDAR, Light Detection And Ranging) were developed for registering optical signals reflected from an atmospheric layer lying at a certain distance. The atmosphere in this case is sounded by short laser impulses and the reflected signal is registered with a certain time resolution. The delay time of the reflected signal determines the distance to the sounded layer. In the absence of natural topographical reflectors (clouds, forest, mountains, buildings), the receiving mirror of the lidar collects reflected laser radiation that has changed upon passing an atmospheric layer. Two lidar versions have been developed, a differential absorption lidar and a Raman effect lidar. The first one is characterized by several orders of magnitude higher sensitivity (or sounding distance) and the second one, which is far behind in sensitivity, possesses a higher selectivity to the chemical composition of the sounded layer. Typical examples of the analytical application of lidars are determinations of the concentrations of $\mathrm{NO}, \mathrm{NO}_{2}, \mathrm{CO}$, $\mathrm{CO}_{2}, \mathrm{O}_{2}$, and other gases in the atmosphere. The determination of ozone in high atmospheric layers by lidars has also been reported.

However, lidars have not received wide acceptance, in spite of the relatively extensive studies in this range and design works on constructing such devices. Much money and efforts has been spent for the development of lidars, and relatively perfect portable complexes have been created. However, in addition to the extremely high cost of the devices and great demands to the staff, the analytical procedure based on lidars has a number of basic limitations. The main limitation is in the non-steady-state atmospheric conditions significantly affecting the propagation of both the sounding and the reflected beams and, therefore, the ambiguity of the extracted information; and also difficulties of signal calibration in terms of the determined concentrations. These serious limitations have not allowed lidars to become a routine tool of analytical control. Although popular scientific journals and sometimes newspapers have readily written about the created lidars, the majority of the solutions has, probably, remained at the level of single specimens used more likely for scientific research rather than for wide analytical application.

In Russia, scientific and engineering works on the development of lidars were performed mainly at the Institute of Atmospheric Optics of the Siberian Division of the Russian Academy of Sciences (Tomsk) and the Institute of Spectroscopy of the Russian Academy of Sciences (Troitsk). A portable experimental complex designed in the 1980s at the Institute of Spectroscopy was used in 1986 in Chernobyl.

What follows from this? Should I write about the lack of prospects for lidars or about the insufficient efforts made to construct these instruments?

The works in this field continue to develop. In 2008, the Koptyug Award of the Siberian Division of the Russian Academy of Sciences was given to researchers from the Zuev Institute of Atmospheric Optics and the Institute of Monitoring of Climatic and Ecological Systems of the Siberian Division of the Russian Academy of Science for a cycle of works "The development of Lidar Methods, Technologies, and Systems for Remote Atmosphere Monitoring for the Lidar Network in the CIS Countries (CIS-LiNET)". The Institute of Atmospheric Optics and its multiple access center (TsKP Atmosfera) actually continues works with lidars, including those for chemical analysis. Single specimens of the developed devices were sold to interested organizations. However, one of the factors hindering the wider use of lidars today is their expensiveness.

Another type of instrument for remote analysis refers to the so-called route measuring instrument. Its operation is based on sounding an unobstructed path with continuous laser radiation and registering radiation reflected either from topographic targets or from a reflector installed at a certain place. In this case, the system measures the average parameters along the path. In the overwhelming majority of cases, an absorption procedure is used. In recent years, instruments of this type have been significantly improved because of the appearance of available short-range and medium IR diode lasers. In a number of megacities, including Moscow, route analyzers of atmospheric pollutants (carbon and nitrogen oxides, etc.) were installed permanently at 
places of traffic congestion for monitoring the level of atmospheric pollution.

Instruments for determining not only atmospheric pollutants, but also compounds important for medical diagnosis, for controlling the storage and application of toxic and hazardous substances, etc. are also being developed. In Russia, such route measuring instruments are developed at the Physical Institute of the
Russian Academy of Sciences and General Physics Institute of the Russian Academy of Sciences.

When writing these notes I used the materials prepared by M.A. Bol'shov and his colleagues at the Institute of Spectroscopy of the Russian Academy of Sciences. I express my sincere gratitude to them.

Yu. A. Zolotov 